\title{
A sequential feature-positive effect using tone as the distinguishing feature in an autoshaping procedure
}

\author{
THOMAS A. LOONEY \\ Lynchburg College, Lynchburg, Virginia 24501 \\ and \\ ROBERT W. GRIFFIN \\ Mercer University, Macon, Georgia 31207
}

\begin{abstract}
Four groups of pigeons were exposed to an autoshaping procedure in which all trials were signaled by a green key light. A tone signaled a food trial for the two tone-positive groups and a no-food trial for the two tone-negative groups. The tone preceded and terminated with green light onset for the two sequential groups and began and ended with the green key light for the two simultaneous groups. The sequential tone-positive group acquired the discrimination faster than the other groups. Asymptotic discrimination was best in the sequential tone-positive and simultaneous tone-negative groups. Relatively few responses occurred on the green key in the simultaneous tone-positive group. The relationship of these results to other studies of the feature-positive effect are discussed.
\end{abstract}

Jenkins and Sainsbury $(1969,1970)$ reported on the asymmetry in the learning of successive go, no-go discriminations that are based on a distinguishing feature. For example, in a study with pigeons, a lighted key was used as an element common to all trials. A distinguishing feature (a black dot) occurred on positive (i.e., reinforced) trials for the featurepositive (FP) group and on negative (i.e., nonreinforced) trials for the feature-negative (FN) group. Discrimination learning was clearly superior for the FP group. Therefore, the asymmetry in discrimination learning has been termed the feature-positive effect (FPE). Although Jenkins and Sainsbury studied the FPE using operant procedures, Hearst (cited in Hearst \& Jenkins, 1974) successfully replicated their findings with a Pavlovian procedure.

Hearst and Jenkins (1974) have proposed an account of the FPE based upon a sign-tracking analysis. They argued that FP superiority can be accounted for on the basis of the degree to which individual elements predict reinforcement. In the FP case, the distinguishing feature is followed by reinforcement on $100 \%$ of its presentations while the common element is followed by reinforcement on

This research was supported in part by Biomedical Support Grant RR07143 from HEW. The authors thank M. A. Looney for her helpful comments, G. C. Scott for his help on the statistical analyses, and M. A. Bennett for typing the manuscript. Reprints may be obtained from T. A. Looney, Department of Psychology, Lynchburg College, Lynchburg, Virginia 24501. The second author is currently at the Gaines Nutrition Center. only $50 \%$ of its presentations. This difference in predictive value results in the subjects' tracking of (i.e., responding to) the distinguishing feature on positive trials. This discrimination between the two elements then leads to a reduction in responding to the common feature during negative trials. In the FN case, however, the distinguishing feature is never followed by reinforcement, whereas the common element is followed by reinforcement on $50 \%$ of the trials. Thus, if a pigeon responds to the most reliable predictor of reinforcement in each case, discriminative performance will be much poorer in the FN condition.

Recently, Morris (1977) has proposed that the effectiveness of a feature in suppressing responding to the common element during FN training depends on the relative size of the feature and the common element. A FPE was found only when a small feature was used in conjunction with a relatively large common element. The effect was not seen when a small feature was used with a small common element or when the large common element was presented with a large feature. Thus, one might conclude that discriminative performance is poor in the $\mathrm{FN}$ case, to the extent that the subject does not receive stimulation from the feature while orienting to the common element.

The present experiment sought to extend previous findings by employing tone as the distinguishing feature and comparing sequential and simultaneous presentation of the feature and common element in 
a Pavlovian procedure. Four groups of pigeons were exposed to an autoshaping procedure in which all trials were signaled by a green key light. A tone signaled a food trial for the two tone-positive groups and a no-food trial for the two tone-negative groups. The tone preceded and terminated with green light onset for the two sequential groups and began and ended with the green key light for the two simultaneous groups.

Some specific predictions about discriminative performance can be made using the conclusions of Hearst and Jenkins (1974) and Morris (1977). In the simultaneous FP condition, the tone was followed by food $100 \%$ of the time while only $50 \%$ of the green light presentations were followed by food. Thus, whether subjects actually tracked the location of the tone (Jenkins in Hearst \& Jenkins, 1974) or engaged in "listening" behavior, or magazineapproach responses as reported by Hearst (in press), one would expect keypecking certainly to be retarded, if, indeed, it occurred at all. On the other hand, the simultaneous FN group would be expected to peck the key because the key light is more predictive of food than the tone. Furthermore, because these subjects cannot avoid being stimulated by the feature while orienting to the common element, they should readily acquire the discrimination. Hence, one would not expect the FPE in the simultaneous conditions.

In the sequential case, responses to the tone are not incompatible with subsequent key responding in the FP group. It is unclear, however, from a strict signtracking account whether subjects will, in fact, peck the key, because the tone predicts the food with certainty while the green light is followed by food only $50 \%$ of the time. One could argue that the feature acquires control over a tendency to respond, and that the green key light following the feature simply offers a locus for the responding to occur. Making that assumption, one might expect stimulus control in the sequential FP group. By comparison, FN subjects would be expected to peck the key because of its predictiveness but to show relatively poor discrimination because they are not exposed to the feature while orienting to the common element. Hence, the FPE might be expected in the sequential conditions.

In addition, Morris' (1977) findings predict that discrimination in the simultaneous FN group would be superior to that of the sequential FN group. The prediction follows from the fact that, in the presence of the common element, the simultaneous FN group cannot avoid being stimulated by the feature, whereas the feature is absent for the sequential FN group.

Finally, a sign-tracking formulation suggests superior discrimination in the sequential FP group relative to the simultaneous FP group due to attenuated responding in the latter condition.

\section{METHOD}

\section{Subjects}

Twenty experimentally naive adult White King pigeons obtained from Palmetto Pigeon Plant were maintained within $15 \mathrm{~g}$ of $75 \%$ of their free-feeding weights. They were housed in individual home cages with health grit and water continuously available under a 16-h-light, 8-h-dark cycle.

\section{Apparatus \\ A $34.9 \times 34.3 \times 33.4 \mathrm{~cm}$ high two-key operant pigeon chamber with a flat-black interior was employed. Only the key on the left side of the front panel was functional; it required a force of approximately $.25 \mathrm{~N}$ to operate. The center of the key was $22.86 \mathrm{~cm}$ from the floor and $11.43 \mathrm{~cm}$ from the nearest wall. At various times, the key was illuminated with either a white or a green light. Food was delivered through a $5.08 \times 5.72 \mathrm{~cm}$ opening, the lower edge of which was $9.53 \mathrm{~cm}$ from the floor. A speaker with a diameter of $6.35 \mathrm{~cm}$ was located on the right side of the panel with its center $11.43 \mathrm{~cm}$ from the floor. The left edge of the speaker was $6.99 \mathrm{~cm}$ from the right side of the food opening. A fan provided both ventilation and masking noise. An Eico (Model 377) audio generator was used to produce a $1,000-\mathrm{Hz}$ square-wave tone. A General Radio Company sound level meter (Type 1565-A) was used to measure sound levels. The microphone was placed in the center of the chamber at the approximate height of a pigeon's head. The sound level was measured to be $86 \mathrm{~dB}$ (A) with the tone and $73 \mathrm{~dB}$ (A) without it. Chamber illumination was provided by a $110-\mathrm{V}$ ac, $7-\mathrm{W}$ shielded light centered on the roof of the chamber. Electromechanical control and recording equipment were located in an adjoining room.}

\section{Procedure}

The subjects were first trained to eat from the food hopper and then exposed to 40 daily sessions of differential autoshaping procedures. Each session consisted of 30 trials. The training procedures for the four groups of birds are shown schematically in Figure 1. All trials included a 5 -sec presentation of a green key light which occurred with an average intertrial interval of $60 \mathrm{sec}$. Half of these trials were followed by $7-\mathrm{sec}$ access to food during which the response key was dark. At all other times, the key was white. A tone was used to signal positive (i.e., food) trials for half of the subjects and negative (i.e., no food) trials for the remaining subjects. In each of these groups, half of the subjects were exposed to a simultaneous (SIM) presentation of the tone and green light, while the remaining subjects were exposed to a sequential (SEQ) condition in which a 5 -sec tone immediately preceded the green light and terminated with green light onset. Thus there were four groups, each having five subjects: SIM tone+, SIM tone-, SEQ tone + , and SEQ tone - . It should be noted that experimental conditions were in no way affected by key responses.

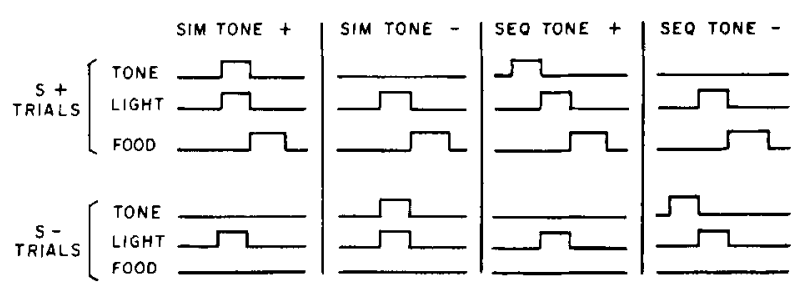

Figure 1. The trial events for the four groups of subjects are shown schematically in time-line format. The groups differ with respect to the tone signaling positive (tone + ) or negative (tone - ) trials and to the tone being presented prior to (SEQ) or concurrent with (SIM) the green key light. 


\section{RESULTS}

Data for three of the four groups (SIM tone + is excluded) are shown in Figure 2. The figure plots mean discrimination ratios. The ratio is of the form $\mathrm{X} /(\mathrm{X}+\mathrm{Y})$, in which $\mathrm{X}$ is the number of responses to the green key on positive trials and $Y$ is the number of responses to the green key on negative trials. A ratio of .5 indicates equal responding to the green key on both positive and negative trials (i.e., no discrimination), and a ratio of 1.0 indicates no responses during negative trials (i.e., perfect discrimination). A discrimination ratio was calculated for each subject and then the mean was calculated from the ratios of all subjects that responded during that session. Once a subject began responding, it responded in all subsequent sessions. In the SEQ tone + group, no birds responded during Session 1 (hence the absence of a point on the graph), four birds responded during Session 2, and all responded during Session 3. In the SEQ tone - group, three, four, and five birds responded during the first, second, and third sessions. In the SIM tone - group, two birds responded during Session 1, four responded during Sessions $2-4$, and the fifth bird began to respond during Session 5 .

The data in the figure were subjected to an analysis of variance which revealed significant effects of Groups $[\mathrm{F}(2,466)=94.22, \mathrm{p}<.001]$, Sessions $[\mathrm{F}(39,466)=$ $17.22, \mathrm{p}<.001]$, and Groups by Sessions $[\mathrm{F}(78,466)$ $=1.48, \mathrm{p}<.05]$. Visual inspection of the figure suggests that the significant interaction was due to an apparent superiority of Group SEQ tone + during early training (i.e., Sessions 1-13) and the lower asymptote of Group SEQ tone - in late training (Sessions 14-40).

To elucidate group differences in acquisition, a comparison was made on the number of sessions to discrimination acquisition. Discrimination was judged to have been acquired when a subject reached a dis-

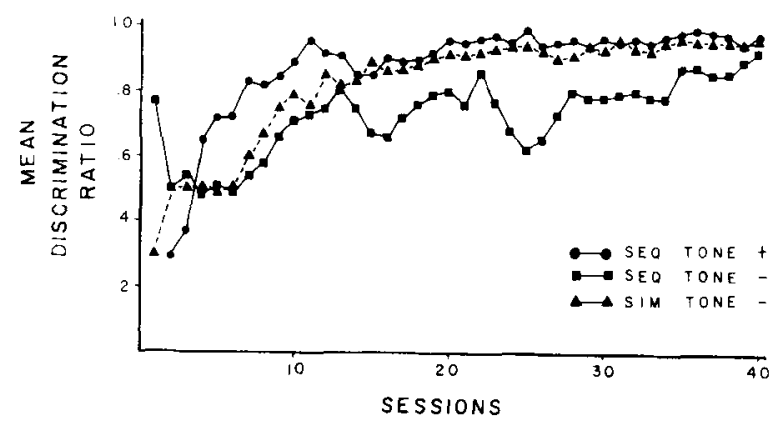

Figure 2. Mean discrimination ratios are shown as a function of sessions for each of three groups. The ratios are of the form $X /(X+Y)$, in which $X$ is the number of responses to the green key on positive trials and $Y$ is the number of responses to the green key on negative trials. crimination ratio of .70 (i.e., approximately twice as many responses on positive trials as on negative trials). Mean number of sessions to reach criterion were $4.8,8.6$, and 7.6 for Groups SEQ tone +, SEQ tone - , and SIM tone-, respectively. SEQ tone + acquired the discrimination significantly earlier than SEQ tone $-[\mathrm{t}(8)=3.80, \mathrm{p}<.01]$ and SIM tone $-[\mathrm{t}(8)=2.37, \mathrm{p}<.05] ;$ SEQ tone - and SIM tone - differences were not significant.

Asymptote was arbitrarily judged to have been reached by Session 14. An analysis of Sessions 14-40 revealed a significant effect of Groups $[F(2,325)=$ $102.33, p<.01]$ and Sessions $[F(26,325)=2.26$, $\mathrm{p}<.01]$. The method of contrasts confirmed the lower level of discrimination in Group SEQ tone relative to Groups SEQ tone $+[F(1,325)=172.04$, $\mathrm{p}<.01]$ and SIM tone $-[\mathrm{F}(1,325)=132.35, \mathrm{p}<.01]$; SEQ tone + and SIM tone - differences were not significant.

Descriptively, acquisition followed similar patterns in the three groups. Responding initially occurred on both positive and negative trials and then continued to increase during positive trials while declining during negative trials. Terminal levels of responding on positive trials were between 150 and 250 responses per session for 14 of the 15 subjects. The one exception was a subject in the SEQ tone + group that usually closed the key switch 25 to 50 times per session. However, observation of this subject revealed that it made many off-key responses which were not recorded. Thus, the positive stimulus may have led to as many pecks by this subject as by the others.

The majority of subjects in the two tone - groups made a substantial number of white-key responses (greater than on positive trials) in at least one session prior to acquisition of the discrimination. In 9 of 10 cases, the level of responding to the white key dropped to that below the level of negative trial responding before stimulus control was gained by the tone. Only one subject in the SEQ tone + group made a substantial number of white-key responses. These responses continued throughout the entire 40 sessions and were generally made in the presence of the tone. Over the last 20 days, approximately 150 responses per session occurred in the tone while about 200 responses occurred to the subsequently presented green key light.

The data for the SIM tone + group were not included in Figure 2 because no responses occurred during many of the sessions, thus resulting in undefined discrimination ratios. The absolute number of responses during the green light is shown in Figure 3 for both positive and negative trials as a function of sessions for three of the subjects. The other two subjects' data are omitted because virtually no keypecks were recorded for the entire 40 sessions. It is obvious that for the three subjects that eventually 


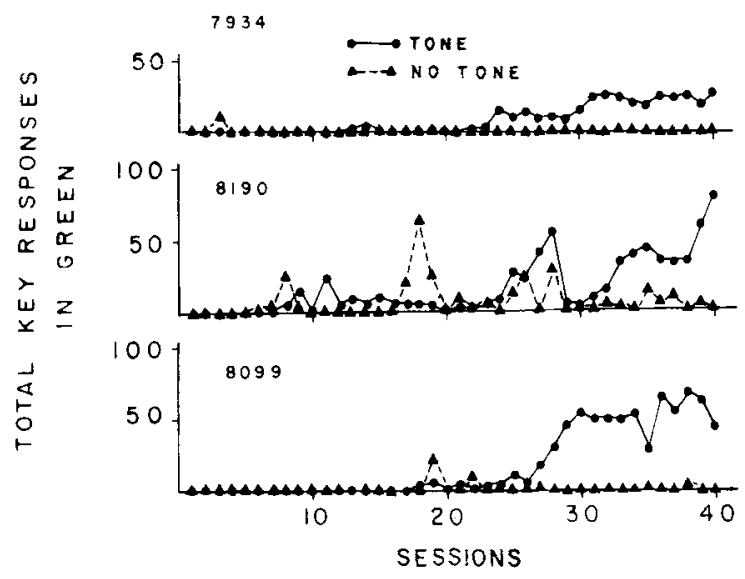

Figure 3. Total key responses during the green light for both positive and negative trials are shown as a function of sessions for three of the subjects in the SIM tone + group.

acquired the discrimination, the absolute level of responding was very low. However, each of the three subjects did respond on the majority of positive trials. Over the final five sessions, Subject 7934 responded on an average of $80 \%$ of the positive trials while Subject 8190 responded on $89 \%$ and Subject 8099 responded on $70 \%$. Unfortunately, these subjects were not observed after acquisition of the discrimination, thus no information concerning off-key pecking exists.

\section{DISCUSSION}

In general, the data of this study support the predictions based on a combination of sign-tracking (Hearst \& Jenkins, 1974) and Morris' (1977) formulation of the FPE. First, in the SIM conditions, discrimination was superior in the $\mathrm{FN}$ group relative to the FP group. It is reasonable to speculate that this result was due to the inability of FN subjects to ignore the feature in the presence of the common element (as predicted by Morris) and due to the severely retarded responding of FP subjects (as predicted by sign-tracking). Indeed, the fact that three of the five FP subjects eventually showed discriminative control by the green light attests to the saliency of that stimulus for pigeons.

The FPE was noted in the SEQ conditions (i.e., performance was superior in the FP condition). This finding is consistent with the results obtained with other types of features (Hearst, in press; Collins, Note 1) and may be accounted for by Morris' (1977) work in that FN subjects can continue to respond to the common element without interference from the feature.

The observation that SIM FN was superior to SEQ FN was expected from Morris' (1977) work insofar as the feature was present to suppress responding in the SIM group but not in the SEQ group.
In contrast to the present results, however, Collins (Note 1) found that the SEQ FN group was clearly superior to the SIM FN group. He, along with previous investigators (e.g., Jenkins \& Sainsbury, 1969), found little evidence for the development of a successive discrimination in the SIM FN group. Thus, one major difference between this and other studies is the present finding that asymptotic discriminative performance in a SIM FN condition is superior to that in the SEQ FN condition. It seems likely that this difference is due, at least in part, to the use of tone as the distinguishing feature in the present study. Perhaps relevant to this issue are the conflicting findings reported by Rescorla (1973) and Collins (Note 1). Both investigators compared the inhibitory control of a negative conditioned stimulus under two conditions. In one condition, the distinguishing feature preceded the common element as in the present study, while in the second condition, the distinguishing feature both preceded and overlapped with presentation of the common feature. Using key lights as the stimuli, Collins found no difference in discrimination learning under these two conditions. Rescorla, on the other hand, used a tone as the distinguishing feature with a light as the common element. He found that when the tone overlapped the light, it developed substantially more inhibitory control than when it simply preceded the light. Rescorla's study did not include a SIM tone - condition, thus preventing direct comparison with the present study; however, his finding does suggest that presentation of the tone in the presence of the light is important for the development of inhibitory control.

In addition, the current study supports the signtracking prediction that SIM FP would be inferior to SEQ FP due to attenuated responding in the SIM group.

One finding in the present work was not expected and is not readily accounted for by Morris' (1977) work. That is, the observation that the SIM FN group acquired the discrimination more slowly than the SEQ FP group does not readily follow from Morris' (1977) analysis. This finding, coupled with previous demonstrations of FPE employing tones as features (Brown, cited in Jenkins \& Sainsbury, 1969, p. 158; Diamond, Goldberg, \& Neff, 1962; Reberg \& Le Clerc, 1977), clearly suggests that Morris' account of the FPE is inadequate.

Similarly, while the sign-tracking account does provide an explanation for some of the data presented herein, Hearst (in press) has noted that a signtracking analysis cannot be readily extended to all situations with diffuse stimuli or the sequential presentation of elements. Additional theories, including the Rescorla and Wagner (1972) model of Pavlovian conditioning (Jenkins, 1973), as well as other accounts 
(see Hearst, in press, for a review) have been used to explain the FPE. No comprehensive theoretical framework currently exists, however, that can predict results from the varicus studies of the FPE which have been conducted with rats, cats, pigeons, and humans. Indeed, Hearst (in press) has argued that until more data are collected, we should consider the possibility that various examples of the FPE are only superficially related and may require separate analyses. Thus, what seem to be called for at this point are additional within-study comparisons of discriminative performance varying the stimuli employed, their intensity, and temporal arrangements.

\section{REFERENCE NOTE}

1. Collins, R. L. Feature negative discrimination in pigeons with simultaneous and sequential compound stimuli. Manuscript submitted for publication.

\section{REFERENCES}

Diamond, I. T., Goldberg, J. M., \& NefF, W. D. Tonal discrimination after ablation of auditory cortex. Journal of Neurophysiology, 1962, 25, 223-235.

Hearst, E. Stimulus relationships and feature selection in learning and behavior. In S. Hulse, H. Fowler, \& W. K. Honig (Eds.), Cognitive processes in animal behavior. Hillsdale, N.J: Earlbaum, in press.
HeARst, E., \& Jenkins, H. M. Sign-tracking: The stimulusreinforcer relation and directed action. Austin Tex: Psychonomic Society, 1974.

JENkINS, H. M. Noticing and responding in a discrimination based on a distinguishing element. Learning and Motivation, 1973, 4, 115-137.

Jenkins, H. M., \& Sainsbury, R. S. The development of stimulus control through differential reinforcement. In N. J. Mackintosh \& W. K. Honig (Eds.), Fundamental issues in associative learning. Halifax: Dalhousie University Press, 1969.

Jenkins, H. M., \& Sainsbury, R. S. Discrimination learning with the distinctive feature on positive or negative trials. In D. Mostofsky (Ed.), Attention: Contemporary theory and anaiysis. New York: Appleton-Century-Crofts, 1970.

MorRIS, R. C. Spatial variables and the feature-positive effect. Learning and Motivation, 1977, 8, 194-212.

Reberg. D., \& LECLERC, R. A feature positive effect in conditioned suppression. Animal Learning \& Behavior, 1977, 5, 143-147.

Rescorta, R. A. Second-order conditioning: Implications for theories of learning. In F. J. McGuigan \& D. B. Lumsden (Eds.), Contemporary approaches to conditioning and learning. Washington, D.C: Winston, 1973.

Rescorla, R. A., \& W AGNER, A. R. A theory of Pavlovian conditioning: Variations in the effectiveness of reinforcement and nonreinforcement. In A. H. Black \& W. F. Prokasy (Eds.), Classical conditioning II: Current research and theory. New York: Appleton-Century-Crofts, 1972.

(Received for publication February 3, 1978; revision accepted May 26, 1978.) 